\title{
Benzodiazepine und Z-Substanzen als Schlaf- und Beruhigungsmittel in einem Krankenhaus
}

\author{
Anwendung aus der Sicht des ärztlichen und pflegerischen Personals
}

\author{
V. Weiß, S. Heinemann, W. Himmel, R. Nau, E. Hummers-Pradier
}

\section{Zusammenfassung}

Hintergrund und Fragestellung I Benzodiazepine und Z-Substanzen kommen als Schlaf- und Beruhigungsmittel häufig zum Einsatz - trotz des langfristig ungünstigen Nutzen-Schaden-Verhältnisses. Ziel dieser Studie war es, herauszufinden, wie häufig diese aus Sicht des Personals in einem Krankenhaus zum Einsatz kommen, wie das Nutzen-Schaden-Verhältnis bei älteren Patienten bewertet wird und welche Faktoren die Einstellung zum Einsatz dieser Medikamente begünstigen.

Methodik I Alle pflegerischen und ärztlichen Mitarbeiter eines Krankenhauses erhielten einen standardisierten Fragebogen zu Einstellungen und Wahrnehmungen im Umgang mit diesen Medikamenten. Einschätzungen über die Häufigkeit der Anwendung und zum Nutzen-SchadenVerhältnis wurden durch Häufigkeitsanalysen ermittelt. Mittels multipler logistischer Regression wurde untersucht, welche Faktoren die Wahrscheinlichkeit erhöhen, dass Benzodiazepine oder Z-Substanzen eingesetzt werden.

Ergebnisse I Deutlich mehr Pflegekräfte als Ärzte glaubten, dass bei Schlafstörungen häufig oder immer Benzodiazepine eingesetzt würden (57\% vs. $29 \%$ ), ebenso bei Z-Substanzen (66\% vs. $29 \%$ ). Fast die Hälfte der Ärzte glaubte, dass Benzodiazepine mehr schaden als nützen, von den Pflegekräften waren es $29 \%$. In der multiplen logistischen Regression hatten folgende Faktoren einen positiven Einfluss auf die wahrgenommene Häufigkeit der Anwendung von Z-Substanzen:

- Zugehörigkeit zum Pflegepersonal (OR: 13,95; 95\%-KI: 3,87-50,28),

- Tätigkeit auf nicht-chirurgischen Abteilungen $(5,41 ; 2,00-14,61)$,

- Berufserfahrung < 5 Jahre $(4,90 ; 1,43-16,81)$,

- die Einschätzung, dass der Nutzen überwiegt $(5,07 ; 1,48-17,35)$.

Im Falle von Benzodiazepinen hatte nur der Nutzenaspekt einen Einfluss auf die wahrgenommene Häufigkeit ihrer Anwendung (3,35; 1,28$8,79)$.

Folgerung I Das ärztliche und pflegerische Personal nimmt die Häufigkeit der Anwendung von Benzodiazepinen und Z-Substanzen unterschiedlich wahr, ebenso das Nutzen-Schaden-Verhältnis. Auch die Tätigkeit auf nicht-chirurgischen Abteilungen oder geringere Berufserfahrung prägen die Einschätzung der Häufigkeit der Anwendung zumindest von Z-Substanzen.

\section{Einleitung}

Zur kurzfristigen symptomatischen Behandlung von Schlafstörungen und Angst- oder Erregungszuständen wird z.B. der Einsatz von Hypnotika wie Benzodiazepine und selektiven Benzodiazepin-Rezeptoragonisten, wie Zolpidem und Zopiclon (kurz Z-Substanzen) empfohlen [1]. Von einer Langzeiteinnahme von $>4$ Wochen wird aufgrund der Nebenwirkungen wie Stürze, kognitive Beeinträchtigungen, Tagesschläfrigkeit, erhöhte Unfallneigung und Abhängigkeit insbesondere bei älteren Menschen abgeraten [15]. Nach San-José et al. (2015) zählen jedoch insbesondere Benzodiazepine gerade bei älteren Patienten zu den am häufigsten im Krankenhaus eingesetzten Medikamenten [6]. Neben der Behandlung von Angst- oder Erregungszuständen finden diese Medikamente in Krankenhäusern auch bei Ein- und Durchschlafstörungen und Schmerzen ihre Anwendung [7]. Bisher lieferte nur die Untersuchung von Somers et al. [7] Ein- blicke in den Umgang mit Schlaf- und Beruhigungsmitteln in einem Krankenhaus: Danach berichteten 54,9\% der 330 befragten Patienten von Ein- und Durchschlafstörungen während eines Krankenhausaufenthaltes. Von diesen 330 Patienten erhielten im Krankenhaus 15,6\% zum ersten Mal ein Medikament aus der Gruppe der Benzodiazepine oder Z-Substanzen, besonders oft auf einer chirurgischen oder rehabilitativen Station.

Für die ambulante Versorgung existieren verschiedene Schätzungen zur Einnahmehäufigkeit und -dauer dieser Medikamente [8-11]. Nach einer Hochrechnung des Norddeutschen Medikamentenmonitors erhalten $4-5 \%$ der gesetzlich Krankenversicherten mindestens eine Verordnung eines Benzodiazepins oder einer Z-Substanz pro Kalenderjahr. Mindestens 1,6 Millionen Menschen in Deutschland nehmen diese länger als sechs Monate ein [11]. 
Von den oben genannten Einzelstudien abgesehen, fehlen verlässliche epidemiologische Daten zur Einnahmehäufigkeit und -dauer von Benzodiazepinen und Z-Substanzen in Krankenhäusern; auch ist nicht bekannt, in welchen Situationen und bei welchen Patienten diese Medikamente eingesetzt werden. Ebenso wenig wissen wir über die Erfahrungen und Wahrnehmungen des ärztlichen und pflegerischen Personals in Krankenhäusern bei der Anwendung dieser Medikamente. Lediglich aus den Ergebnissen einer Fokusgruppen-Studie mit Pflegekräften in belgischen Altenpflegeheimen könnte man vermuten, dass sich der Einsatz von Schlaf- und Beruhigungsmitteln manchmal als einfache und risikoarme Lösung zur Aufrechterhaltung des Schlaf-Wach-Rhythmus der Heimbewohner anbietet, aber schnell zum Dauergebrauch führt [13]. Im Vordergrund unserer Studie standen daher folgende Fragen:

1. Wie beurteilt das pflegerische und ärztliche Personal eines Krankenhauses die Verwendung von Benzodiazepinen und Z-Substanzen?

2. Wie bewerten beide Gruppen das Nutzenund Schadenverhältnis dieser Medikamente bei älteren Patienten?

3. Welche Faktoren begünstigen bzw. beeinflussen eine positive Einstellung/Wahrnehmung dieser Medikamente in einem Krankenhaus?

\section{Methode}

Design | Diese Querschnittstudie fand in einem Krankenhaus der Grund- und Regelversorgung mit insgesamt 342 vollstationären Betten statt im Rahmen eines Forschungsprojekts zur Verordnungen von Benzodiazepinen und Z-Substanzen an der Schnittstelle von Krankenhaus und Hausarzt [24]. Alle Angehörige des pflegerischen Personals (mit Ausnahme von Auszubildenden) und ärztlichen Personals erhielten einen standardisierten Fragebogen zur Anwendung von Benzo-

Tab. 1 Stichproben (alle Angaben in Prozent)

\section{ärztliches Personal ( $n=63) \quad$ Pflegepersonal $(n=68)$}

\section{Geschlecht}

- Männer

65,1

17,7

- Frauen

34,9

82,3

\section{Abteilung}

- chirurgisch

- nicht-chirurgisch

\section{Dauer der Berufstätigkeit}

- < 5 Jahre

47,6

30,2

22,2
23,5

20,6

55,9 diazepinen und Z-Substanzen als Schlaf- und Beruhigungsmittel. Um eine möglichst hohe Rücklaufquote zu erreichen, übernahmen wir folgende Empfehlungen aus einem aktuellen Cochrane Review [13]: Ankündigung der Studie in der Mitarbeiterzeitschrift des Krankenhauses, persönliche Kontaktaufnahme zum ärztlichen und pflegerischen Personal auf den Stationen, einladende Gestaltung des Briefumschlages sowie zusätzliche Informationen zur Studie durch einen Flyer (mit Hinweisen zum Datenschutz, Ziele der Untersuchung und Ansprechpartner). Nach acht Wochen wurde noch einmal an die Befragung erinnert. Es gab keine finanziellen Anreize, an der Studie teilzunehmen.

Fragebogen | Für die standardisierte Befragung wurde der Fragebogen von Hoffmann verwendet [14]. Ursprünglich richtete er sich an Hausärzte, um Einstellungen zu Risiken und Nebenwirkungen von Benzodiazepinen und Z-Substanzen zu erfragen. Der Fragebogen wurde für die stationäre Versorgungssituation angepasst. Beide Berufsgruppen erhielten identische Fragen und wurden nach ihrem individuellen Anwendungsverhalten gefragt. Da Pflegekräfte Medikamente nicht verordnen dürfen, wurden sie um eine Einschätzung der Verordnungshäufigkeit bzw. Verwendung gefragt.

Der Fragebogen umfasste folgende Themen:

- medikamentöse und nicht-medikamentöse Interventionen bei Schlafstörungen und Angst-, Anspannungs- oder Erregungszuständen

- Wirksamkeit und unerwünschte Wirkungen von Benzodiazepinen und Z-Substanzen

- Nutzen-Schaden-Verhältnis dieser Medikamente

- personenbezogene Angaben (Geschlecht, Tätigkeit, Abteilung)

Im Rahmen des o.g. Forschungsprojektes stand die Anwendung von Benzodiazepinen und Z-Substanzen als Schlaf- und Beruhigungsmittel im Vordergrund.

Die Einschätzung zur Häufigkeit der Anwendung der Medikamente wurde durch eine 5-stufige Skala ermittelt. Auch die Einschätzung des Nutzen-Schaden-Verhältnisses erfolgte fünfstufig mit den Extremen: „Nutzen überwiegt deutlich“ und „Schaden überwiegt deutlich“.

Datenauswertung I Die Auswertung zur Häufigkeit der Anwendung und zum Nutzen-SchadenVerhältnis der Medikamente erfolgte deskriptiv (absolute und relative Häufigkeiten). Einflussfaktoren für die wahrgenommene Häufigkeit der Verwendung von Benzodiazepinen oder Z-Substanzen wurden mittels multipler logistischer Regression bestimmt - mit Odds Ratios und ihren 95\%-Konfidenzintervallen als Effektgrößen. Antworten auf die Frage nach der Häufigkeit der Anwendung von Benzodiazepinen oder Z-Substan- 


\begin{tabular}{|lllll|}
$\begin{array}{l}\text { Häufigkeit der } \\
\text { Anwendung* }\end{array}$ & $\begin{array}{l}\text { Benzodiazepine } \\
\text { ärztliches Personal } \\
(\mathbf{n = 6 3 )}\end{array}$ & $\begin{array}{l}\text { Pflegepersonal } \\
\mathbf{( n = 6 8 )}\end{array}$ & $\begin{array}{l}\text { ärztliches Personal } \\
\mathbf{( n = 6 3 )}\end{array}$ & $\begin{array}{l}\text { Pflegepersonal } \\
(\mathbf{n = 6 8 )}\end{array}$ \\
\hline gar nicht & 17,5 & 0,0 & 20,6 & 0,0 \\
\hline selten & 31,8 & 7,4 & 19,1 & 8,8 \\
manchmal & 22,2 & 35,3 & 31,8 & 25,0 \\
\hline häufig & 28,6 & 51,5 & 28,6 & 64,7 \\
\hline immer & 0,0 & 5,9 & 0,0 & 1,5
\end{tabular}

* nach Einschätzung der Befragten; alle Angaben in \%

zen wurden - soweit sinnvoll - dichotomisiert: „nicht häufig“ (gar nicht, selten, manchmal) vs. „häufig“ (häufig und immer). Einflussfaktoren im Modell waren:

- Geschlecht

- Beruf(Arzt, Pflegekraft)

- Abteilung (chirurgische, nicht-chirurgische Abteilung)

- Dauer der beruflichen Tätigkeit im betreffenden Krankenhaus (>10 Jahre, 5-10 Jahre, <5 Jahre)

- die Einschätzung des Nutzen-Schaden-Verhältnisses [15]

Alle Berechnungen erfolgten mittels SAS 9.4 für Windows.

Einhaltung ethischer Richtlinien | Die Ethik-Kommission der Universitätsmedizin Göttingen stufte die Untersuchung als ethisch unbedenklich ein (25/2/14). Auch die Mitarbeitervertretung des Krankenhauses gab für die Befragung ihr Einverständnis. Das ärztliche und pflegerische Personal wurde ausdrücklich darüber informiert, dass eine Teilnahme an der Studie freiwillig ist. Die ausgefüllten Fragebögen und unterschriebenen Einverständniserklärungen gaben die Teilnehmer getrennt voneinander in geschlossenen Briefumschlägen $a b$.

\section{Ergebnisse}

Teilnehmer I Insgesamt beantworteten 138 von 359 Mitarbeitern (38\%) den Fragebogen, deutlich häufiger ärztliche Mitarbeiter (65/126; 51\%) als pflegerische Mitarbeiter (73/282; 26\%).

In die Auswertung kamen nur Fragebögen mit vollständigen Angaben zu Geschlecht, Abteilung, Dauer der beruflichen Stellung und zur Einschätzung der Häufigkeit der Verwendung von Benzodiazepinen oder Z-Substanzen $(n=131)$. Bei den ärztlichen Mitarbeitern dominierten die Männer (65\%), umgekehrt war es beim Pflegepersonal (18\%). Etwa ein Drittel aller befragten Krankenhausmitarbeiter war auf chirurgischen Stationen tätig. Das Pflegepersonal war deutlich berufserfahrener als das ärztliche Personal ( Tab. 1).

\section{Einschätzung zur Verwendung von Benzodiazepi-} nen und Z-Substanzen | Bezogen auf die jeweiligen Stationen schätzte das Pflegepersonal die Häufigkeit der Verwendung der beiden Medikamentengruppen deutlich höher ein als das ärztliche Personal ( Tab. 2). Das galt sowohl für Benzodiazepine als auch für Z-Substanzen. Weit über die Hälfte des befragten Pflegepersonals berichtete, dass Schlafmittel bei Schlafstörungen häufig verwendet werden. Beim ärztlichen Personal berichtete dies nur ein knappes Drittel. Dass diese Medikamente gar nicht bei Schlafstörungen eingesetzt werden, meinten ca. $20 \%$ der Ärzte, niemand dagegen vom Pflegepersonal.

Bewertung des Nutzen-Schaden-Verhältnisses von Benzodiazepinen und Z-Substanzen I Fast die Hälfte des ärztlichen Personals war der Ansicht, dass der Schaden bei Benzodiazepinen den Nutzen bei älteren Patienten überwiegt (48\%), selte-
Tab. 2 Geschätzte Häufigkeit der Anwendung von Benzodiazepinen und Z-Substanzen bei Schlafstörungen.
Tab. 3 Nutzen-SchadenVerhältnis beider Medikamentengruppen bei älteren Patienten.

\begin{tabular}{|lllll}
\hline Nutzen-Schaden-Verhältnis* & $\begin{array}{l}\text { Benzodiazepine } \\
\text { ärztliches Personal } \\
(\mathbf{n = 6 1 )}\end{array}$ & $\begin{array}{l}\text { Pflegepersonal } \\
\mathbf{( n = 6 8 )}\end{array}$ & $\begin{array}{l}\text { Z-Substanzen } \\
\text { ärztliches Personal } \\
(\mathbf{n = 5 5 )}\end{array}$ & $\begin{array}{l}\text { Pflegepersonal } \\
\text { (n=67) }\end{array}$ \\
\hline Nutzen überwiegt & 19,7 & 39,7 & 30,9 & 44,8 \\
\hline halten sich die Waage & 32,8 & 30,9 & 40,0 & 37,3 \\
\hline Schaden überwiegt & 47,5 & 29,4 & 29,1 & 17,9 \\
\hline * nach Einschätzung der Befragten; alle Angaben in \% & & &
\end{tabular}


Benzodiazepine

OR
95\%-Konfidenzintervall P

\section{Z-Substanzen}

OR
95\%-Konfidenzintervall P

\section{Geschlecht}

- Frau

- Mann

Beruf

- ärztliches Personal

- Pflegepersonal

Abteilung

- chirurgische Abteilung

- nicht-chirurgische Abteilung

\section{Dauer der Berufstätigkeit}

- >10 Jahre

- 5-10 Jahre

- <5 Jahre

Nutzen-Schaden-Verhältnis

- Schaden überwiegt

- halten sich die Waage

- Nutzen überwiegt
1,00

1,19

1,00

2,48

$0,96-6,36$

1,00

0,66

$0,30-1,46$

0,3002

1,00

5,41

$2,00-14,61$

0,0009
1,00

0,74

0,47

0,28-1,99

$0,18-1,19$

1,00

2,36

3,35
0,94-5,94

$1,28-8,79$
1,00

$0,5557 \quad \mathbf{4 , 0 2}$

$1,17-13,88$

0,0277

$0,1118 \quad \mathbf{4 , 9 0}$

$1,43-16,81$

0,0115

1,00

$0,0678 \quad 3,96$

0,0141
$1,18-13,32$
$1,48-17,35$

0,0262

0,0096

Tab. 4 Einflussfaktoren für die Einschätzung einer häufigen Anwendung von Benzodiazepinen und Z-Substanzen als Schlaf- und Beruhigungsmittel. Aufgrund vereinzelter Ausfälle bei den Fragen zum Nutzen-Schaden-Verhältnis reduzierte sich die Stichprobe auf N=130 (Benzodiazepine) bzw. $\mathrm{N}=122$ (Z-Substanzen). OR = Odds ratio (signifikante Werte sind fett markiert)

ner war das Pflegepersonal dieser Meinung (29\%). Bzgl. der Z-Substanzen glaubten dies 29\% der Ärzte und $18 \%$ der Pflegekräfte ( Tab. 3). Entsprechend häufiger als Ärzte glaubte das Pflegepersonal, dass der Nutzen von Benzodiazepinen den möglichen Schaden überwiegt (40\% vs. $20 \%$ ), ähnlich bei Z-Substanzen.

Was beeinflusst die Einschätzung der Häufigkeit der Anwendung von Benzodiazepinen und Z-Substanzen? | Für die Einschätzung, dass Benzodiazepine oder Z-Substanzen häufig verwendet werden, hatte im multivariaten Modell das vermutete Nutzen-Schaden-Verhältnis einen signifikanten Einfluss ( $\bullet$ Tab. 4): Wenn aus Sicht der Befragten der Nutzen von Benzodiazepinen deren potenziellen Schaden überwog, waren sie vergleichsweise oft der Ansicht, diese Medikamente häufig zu verwenden bzw. zu verordnen (Odds Ratio: 3,35; 95\%-Konfidenzintervall: 1,28$8,79)$. Dieser Zusammenhang galt auch für Z-Substanzen $(5,07 ; 1,48-17,35)$. Anders als bei den Benzodiazepinen spielten bei den Z-Substanzen aber noch weitere Faktoren eine signifikante Rolle, insbesondere die Berufszugehörigkeit. So waren deutlich mehr Mitarbeiter aus der Pflege als Ärzte der Ansicht, Z-Substanzen als Schlaf- und Beruhigungsmittel häufig zu verwenden (13,95;
3,87-50,28). Auch die Tätigkeit auf nicht-chirurgischen Abteilungen (im Vergleich zu anderen Abteilungen), eine kürzere Berufstätigkeit und die Einschätzung, dass bei Z-Substanzen das Nutzen-Schaden-Verhältnis ausgeglichen ist bzw. der Nutzen überwiegt, waren in diesem Zusammenhang von Bedeutung.

\section{Diskussion}

Mitarbeiter des ärztlichen und pflegerischen Personals nehmen die Häufigkeit der Verwendung von Benzodiazepinen und Z-Substanzen unterschiedlich wahr, ebenso das Nutzen-SchadenVerhältnis dieser Medikamente. Vermuteten Mitarbeiter in der Anwendung von Benzodiazepinen einen höheren Nutzen als Schaden, insbesondere bei älteren Patienten, waren sie auch vergleichsweise oft der Ansicht, diese Medikamente häufiger anzuwenden. Dies galt auch für die häufigere Anwendung von Z-Substanzen. Hier spielten aber zusätzlich die Tätigkeit als Pflegekraft, die Arbeit auf nicht-chirurgischen Abteilungen oder auch geringere Berufserfahrung eine Rolle.

Verglichen mit Ärzten, gaben doppelt so viele Mitarbeiter aus der Pflege an, Benzodiazepine 
und Z-Substanzen häufig zu verwenden - das attestiert dieser Berufsgruppe eine nicht zu unterschätzende Rolle im Umgang mit Schlaf- und Beruhigungsmitteln. Im Gegensatz zum ärztlichen Personal verbringt das pflegerische Personal deutlich mehr Zeit mit den Patienten [16, 17]. Es ist zu vermuten, dass Pflegekräfte oft näher am Patienten sind, erste Ansprechpartner bei Einund Durchschlafstörungen sind und auch die Verordnung bzw. Gabe von Bedarfsmedikamenten veranlassen. Demzufolge könnte die Einschätzung der Pflegekräfte zutreffender als die der Ärzte sein und die Ausgabe von Benzodiazepinen und Z-Substanzen tatsächlich regelmäßig und häufiger erfolgen, als Ärzte vermuten.

Wie in Hoffmanns Befragung von Hausärzten [14], sahen auch in unserer Studie viele Ärzte (31\%) bei Z-Substanzen einen größeren Nutzen als Schaden, gerade für ältere Patienten. Allerdings meinten $40 \%$ der befragten Ärzte, dass sich Nutzen und Schaden die Waage hielten. Das Pflegepersonal schätzte die Bilanz positiver ein: Knapp 40\% bzw. knapp 45\% meinten, dass bei älteren Patienten der Nutzen von Benzodiazepinen bzw. Z-Substanzen den Schaden überwiegt. Nach den vorhandenen und auch für Pflegekräfte zur Verfügung stehenden Leit- und Richtlinien wie bspw. der im kooperierenden Krankenhaus berücksichtigten PRISCUS-Liste sollte jedoch bekannt sein, dass beide Medikamentengruppen bei älteren Patienten mit Begleiterkrankungen wie Demenz, Ateminsuffizienz oder einer bekannten Gefährdung für Substanzabhängigkeit nur unter engmaschiger klinischer Kontrolle eingesetzt werden sollten, um Nebenwirkungen rechtzeitig erkennen zu können. Der in unserer Studie - wie auch in der Studie von Anthierens et al. (2009) [12] - insbesondere von den Pflegekräften wahrgenommene großzügige Einsatz beider Medikamentengruppen widerspricht diesen Empfehlungen.

Z-Substanzen kommen - insbesondere in der Wahrnehmung der Pflegekräfte - häufiger zum Einsatz als Benzodiazepine. Beide Berufsgruppen attestieren ihnen auch das bessere Nutzen-Schaden-Verhältnis. Möglicherweise drückt sich hierin der Therapie-Optimismus aus, der die Einführung der Z-Substanzen begleitete. So empfahl z.B. Antai-Otong (2006) in ihrem Review für Pflegekräfte in der Psychiatrie die Z-Substanzen wegen ihrer angeblichen Sicherheit, Wirksamkeit und geringeren Nebenwirkungen als gute Option bei Schlafproblemen [18]. Dazu passt auch unser Ergebnis, dass Ärzte und Pflegekräfte, deren Ausbildung noch nicht so lang zurückliegt, von einer deutlich häufigeren Anwendung dieser Substanzen berichten. Auch wenn mittlerweile in der wissenschaftlichen Diskussion die Euphorie der realistischen Einschätzung gewichen ist, dass ZSubstanzen nicht minder riskant hinsichtlich Stürzen und kognitiver Beeinträchtigungen am
Morgen sind [19], wurden sie aber kürzlich noch in der auflagenstarken Streuzeitung „Der Privatarzt“" als ideales Schlafmittel“ angepriesen [20]. Der Nimbus wirkt offensichtlich fort.

Krankenhausaufenthalte - und die damit einhergehenden Akutsituationen - können ein potenzieller Ort für den (erstmaligen) Einsatz von Benzodiazepinen und Z-Substanzen sein und zeitgleich als Initiator für Folgeverordnungen fungieren, wenn auch nicht unbedingt für eine gefährliche Langzeiteinnahme [21]. Dass ein Krankenhausaufenthalt die weiterführende (bspw. hausärztliche) Medikation beeinflussen kann, ist aus anderen Untersuchungen bekannt [22]. Die recht großzügige angenommene Verwendung von Benzodiazepinen und Z-Substanzen, wie sie zumindest Pflegekräfte in unserer Untersuchung berichteten, könnte die Grundlage für einen Fortsetzungswunsch dieser Medikamente legen. Zukünftige Studien sollten den Umgang mit Benzodiazepinen und Z-Substanzen in Krankenhäusern - als eventuellen Ausgangspunkt für eine erstmalige Verordnung von Hypnotika - weiter fokussieren [7].

Die aus der Befragung sich abzeichnenden unterschiedlichen Einschätzungen des ärztlichen und pflegerischen Personals legen es nahe, beide Berufsgruppen über einen adäquaten Umgang von Benzodiazepinen und Z-Substanzen und über die Risiken einer Weiterverordnung im ambulanten Sektor zu informieren bzw. fortzubilden, möglichst interdisziplinär, um zur gemeinsamen Entwicklung von Lösungsstrategien zu kommen [23]. Dies wäre auch ein Thema für Arzneimittelkommissionen, soweit in Krankenhäusern ansässig.

Stärken und Schwächen | Die Untersuchung erlaubt erstmals Einblicke zur Einschätzung der Anwendung von Benzodiazepinen und Z-Substanzen und vor allem zu den Einstellungen des pflegerischen und ärztlichen Personals eines Krankenhauses der Grund- und Regelversorgung gegenüber diesen Medikamenten. Gerade der Vergleich beider Berufsgruppen zeigt Ansatzpunkte für Interventionen.

Trotz intensiver Planung und vorbereitender Maßnahmen [14] ist die Beteiligung des Pflegepersonals an der Befragung mit 26\% enttäuschend niedrig, ein Selektionsbias kann nicht ausgeschlossen werden. Die Länge des Fragebogens könnte ein Grund für die Nichtteilnahme gewesen sein, aber auch Unsicherheiten in der Anwendung der Medikamente, die man ungern artikulieren wollte. Beide Berufsgruppen hatten vereinzelt Schwierigkeiten, die beiden Medikamentengruppen getrennt voneinander zu bewerten, wie sich z.B. aus den fehlenden Antworten zur Einschätzung der Häufigkeit der Anwendung und des Nutzen-Schaden-Verhältnisses andeutet. Neben möglichen Schwächen des Fragebogens 


\section{Danksagung}

Die Studie wird durch das Bundesministerium für Gesundheit finanziert (Förderkennzeichen: IIA5-2513DSM228). Wir danken allen Ärzten, Pflegekräften, Mitarbeitern der Personalabteilung und der Mitarbeitervertretung des Ev. Krankenhaus Göttingen-Weende für die Zusammenarbeit. Unser besonderer Dank gilt dem Ärztlichen Direktor Prof. Dr. Michael Karaus und dem kaufmännischen Geschäftsführer Frank Czeczelski. Nur durch ihre Unterstützung konnte die Studie durchgeführt werden. Prof. Dr. Falk Hoffmann, Universität Anteil an dieser Studie und dem Artikel. Kollegial überließ er uns seinen Fragebogen und erlaubte die Anpassung an die Krankenhaussituation. Mit großem Gewinn übernahmen wir seine konstruktiven Vorversion des Manuskripts. Oldenburg, hat besonderen Änderungsvorschläge einer

unterstreicht dieser Mangel die Dringlichkeit von Fortbildungen, um defizitäre pharmakologische Kenntnisse auszugleichen.

Eine weitere Schwäche ist die Beschränkung auf subjektive Einschätzungen und Wahrnehmungen zur Anwendung von Benzodiazepinen und ZSubstanzen. In einem nächsten Schritt soll durch eine retrospektive Sichtung von Patientenakten (Chart-Review) das tatsächliche Verordnungsgeschehen - als Bedarfs- und Dauermedikation erhoben werden, einschließlich der dann im Entlassungsbrief empfohlenen Medikamente. Des Weiteren stehen noch qualitative Interviews mit dem ärztlichen und pflegerischen Personal aus, um die vorliegenden Ergebnisse besser einordnen zu können.

\section{Konsequenz für Klinik und Praxis}

- Die befragten Berufsgruppen schätzen die Häufigkeit der Anwendung von Benzodiazepinen und Z-Substanzen unterschiedlich ein.

- Z-Substanzen haben gegenüber Benzodiazepinen eine höhere Akzeptanz, sowohl beim ärztlichen als auch pflegerischen Personal, wenngleich die wissenschaftliche Evidenz fehlt.

- Die Faktoren für die wahrgenommene Häufigkeit der Medikamentenanwendung wie z.B. Annahmen über das NutzenSchaden-Verhältnis, die Tätigkeit als Pflegekraft oder geringere Berufserfahrung können Interventionen zur Verbesserung des Umganges mit Schlaf- und Beruhigungsmitteln in Krankenhäusern anregen.

\section{Literatur}

1 National Institute for Health and Care Excellence (NICE). Guidance on the use of zaleplon, zolpidem and zopiclone for the short-term management of insomnia. https://www.nice.org.uk/guidance/ta77/ chapter/about-this-guidance. Letzter Zugriff: 17.11.2015

2 Jahnsen K, Roser P, Hoffmann K. Probleme der Dauertherapie mit Benzodiazepinen und verwandten Substanzen. Dtsch Arztbl 2015; 112: 1-7

3 Olfson M, King M, Schoenbaum M. Benzodiazepine Use in the United States. JAMA psychiatry 2014: E1-E7

4 Holt S, Schmiedl S, Thürmann P. Potentially inappropriate medications in the elederly: the PRISCUS list. Dtsch Arztbl Int 2010; 107: 543-551

5 Billioti de Gage, Sophie, Bégaud B et al. Benzodiazepine use and risk of dementia: prospective population based study. BM] 2012; 345: e6231

Interessenkonflikt Die Autoren geben an, dass kein Interessenkonflikt besteht.

DOI $10.1055 / \mathrm{s}-0042-102618$ Dtsch Med Wochenschr 2016; 141: e121-e126 (c) Georg Thieme Verlag KG . Stuttgart · New York . ISSN 0012-0472
8 Glaeske G. Psychotrope und andere Arzneimittel mit Missbrauchs- und Abhängigkeitspotential. In: Deutsche Hauptstelle für Suchtfragen e. V., Hrsg. Jahrbuch Sucht 2006. Geesthacht: Neuland Verlagsgesellschaft; 2006: 87-103

9 Schwabe U, Paffrath D, Hrsg. ArzneiverordnungsReport 2015. Aktuelle Daten, Kosten, Trends und Kommentare. Berlin, Heidelberg: Springer; 2015

10 Verthein U, Martens MS, Raschke P et al. Langzeitverschreibung von Benzodiazepinen und NonBenzodiazepinen. Gesundheitswesen 2013; 75: 430-437

11 Holzbach R, Martens M, Kalke J et al. Zusammenhang zwischen Verschreibungsverhalten der Ärzte und Medikamentenabhängigkeit ihrer Patienten. Bundesgesundheitsbl 2010; 53: 319-325

12 Anthierens S, Grypdonck M, Pauw L de et al. Perceptions of nurses in nursing homes on the usage of benzodiazepines. J Clin Nurs 2009; 18: 3098-3106

13 Edwards PJ, Roberts I, Clarke MJ et al. Methods to increase response to postal and electronic questionnaires . Cochrane Database Syst Rev 2009; 8

14 Hoffmann F. Perceptions of German GPs on benefits and risks of benzodiazepines and Z-drugs. Swiss Med Wkly 2013; 1-7

15 Stokes ME, Davis C, Koch G. Categorical data analysis using the SAS system. 3. Aufl. Cary. NC: SAS Institute; 2012

16 Weigl M, Müller A, Zupanc A et al. Participant observation of time allocation, direct patient contact and simultaneous activities in hospital physicians. BMC health 2009; 9: 110

17 O'Leary KJ, Liebovitz DM, Baker DW. How hospitalists spend their time: insights on effi-ciency and safety. J Hosp Med 2006; 1: 88-93

18 Antai-Otong D. The art of presricbing. Risks and benefits of non-benzodiazepine receptor agonists in the treatment of acute primary insomnia in older adults. Perspect Psychiatr Care 2006; 42: 196-200

19 Gunja N. In the Zzz zone: the effects of Z-drugs on human performance and driving. J Med Toxicol 2013; 9: 163-171

20 Hajak G. Das ideale Schlafmittel lässt gut schlafen und fit aufwachen. Privatarzt 2014; 18

21 Stuffken R, van Hulten R, Rolf P et al. The impact of hospitalisation on the initiation and long-term use of benzodiazepines. Eur J Clin Pharmacol 2006; 61: 291-295

22 Himmel W, Kochen MM, Sorns U et al. Drug changes at the interface between primary and secondary care. Int J Clin Pharm Ther 2004; 42 : 103-109

23 Zwarenstein M, Reeves S, Barr H et al. Interprofessional education: effects on professional practice and health care outcomes. Cochrane Database Syst Rev 2001: 1

24 Heinemann S, Weiß V, Straube K et al. Understanding and reducing the prescription of hypnotics and sedatives at the interface of hospital care and general practice: A protocol for a mixed-methods study. BM] Open 2016 [in press] 